\title{
Nurses' Caring Attitude: Fall Prevention Program Implementation as an Example of Its Importance
}

\author{
Huey-Ming Tzeng, PhD, RN \\ Huey-Ming Tzeng, PhD, RN, is Professor and Associate Director of Nursing and Undergraduate Programs, Department \\ of Nursing, School of Health Professions and Studies, The University of Michigan-Flint, Flint, MI.
}

\author{
Keywords \\ Accidental fall, attitude, attitude of \\ health personnel, hospitals, \\ inpatients, inservice training, \\ knowledge, patient-centered care, \\ quality of health care, safety

\section{Correspondence} \\ Huey-Ming Tzeng, PhD, RN, \\ Department of Nursing, School of \\ Health Professions and Studies, \\ The University of Michigan-Flint, \\ 303 E. Kearsley Street, 2180 WS \\ White Building, Flint, Ml \\ 48502-1950. \\ E-mail: tzenghm@gmail.com
}

BACKGROUND OF THE PROBLEM. Fall prevention programs are universally multidisciplinary, but nursing care plays the central role. Since October 2008, Medicare has no longer reimbursed acute care hospitals for the costs of additional care required due to hospital-acquired injuries (e.g., injurious falls).

PROBLEM. However, fall prevention programs for hospitalized patients have had limited success, and multifaceted strategies for implementing fall prevention programs cannot guarantee success. It is possible that cultivating and sustaining a caring attitude among clinicians is often overlooked as an intervention strategy.

METHOD. This article discusses the barriers to implementing fall prevention programs in acute care hospitals. The attributional theory of success and failure is used to analyze these barriers. In addition, the author discusses whether a lack of knowledge and/or a lack of caring attitude play a role as the underlying barriers to implementing a successful fall prevention program. A patient's story illustrates patients' expectations for the care environment to center on their needs. Possible educational strategies as interventions for fall prevention programs are discussed.

FINDINGS. It is suggested that education goals for nurses need to not only promote their professional knowledge and skills in implementing a fall prevention program but also cultivate their caring attitudes.

\section{Introduction}

Fall prevention programs are universally multidisciplinary, but nursing centered (Gutierrez \& Smith, 2008). Falls are multifactorial in nature and associated with multiple medical, functional, and cognitive factors (Gilewski, Roberts, Hirata, \& Riggs, 2007; Joint Commission, 2005; Schwendimann, Bühler, De Geest, \& Milisen, 2006; Tinetti, Inouye, Gill, \& Doucette, 1995; Tinetti \& Kumar, 2010; Tinetti \& Williams, 1997). Factors and situations that increase the risk of falls and fall-related injuries include unsafe gait and transfer, difficulty in vision and visual perception, unsafe personal care (e.g., risk-taking behaviors in toileting and use of assistive device), cognitive impairment and difficulty in understanding or following instructions, incontinence, medical conditions and related medication use, nutrition status, and environment (e.g., lighting, difficulty in using the call light, staffing, and delayed care) (Aberg, Lundin-Olsson, \& Rosendahl, 2009; Currie, 2008; Joint Commission, 2005; Quigley et al., 2009; Tzeng \& Yin, 2008a). Estimates of inpatient falls during hospital stays have been constant over time (2.2-7 per 1,000 patient-days). About 30\% of patients who fall sustain physical injury leading to longer lengths of stay and higher hospital costs (Hitcho et al., 2004; Nadkarni, Iyengar, Dussa, Watwe, \& Vishwanath, 2005; Rizzo et al., 1998).

Nursing would be best positioned to influence the healthcare system and practice if it combined its quest 
for holistic and patient-centered care with sciencebased advocacy (Aiken, 2008). Bedside nurses' opinions of effective fall prevention measures show the importance of providing patient-centered care with attention to each individual patient's needs (e.g., ensuring a patient is treated for pain or agitation) and being proactive (e.g., anticipating patients' needs, assisting patients to get up or sit if they are able) (Gutierrez \& Smith, 2008). However, fall prevention programs for hospitalized patients have had limited success.

For example, in a study conducted by Raeder, Siegmund, Grittner, Dassen, and Heinze (2010) in 28 German hospitals, patients in hospitals with guidelines were surprisingly more likely to fall than patients in hospitals without guidelines or with guidelines still in development. However, when the inpatient care unit and individual patient variables were included in multilevel regression analyses (hospital and inpatient care unit levels of data were used), the use of fall prevention guidelines was statistically significant in reducing the frequency of fall-related injuries (Raeder et al., 2010). The effect of program implementation on a decrease in total falls and fall-related injuries is still inconclusive.

Would appropriate plans of care for patient monitoring and surveillance be a cause of patient falls? For example, the study conducted by Tzeng, Yin, and Grunawalt (2008) in an acute care setting in Michigan found that if the number of sitter requests was higher, the total number of restraints would be lower but the total fall rate would be higher. Another study conducted by Tzeng and Yin (2009) in a medical center in Taiwan found that if no family members were present when a patient fell, the fall-related injury was less serious. The results suggested that nurses in Taiwan tend to depend on family members to help provide patient care. In other words, the answer to the aforementioned question is that the effect of patient monitoring and surveillance on a decrease in inpatient falls is also still inconclusive.

In a study conducted by Koh, Hafizah, Lee, Loo, and Muthu (2009) in two acute care hospitals in Singapore, a multifaceted strategy for implementing a fall prevention program (i.e., revision of fall prevention policies, development of a quick reference guide on fall prevention, use of change champions and education sessions, adoption of reminders and identification systems, use of audit and feedback methods) was found to effectively increase nurses' knowledge and their compliance with fall risk assessment. The educa- tion sessions included discussion of the importance of fall prevention, identification of fall risk factors by using a risk assessment tool, skills required to conduct a fall risk assessment, and interventions for preventing falls. However, this multifaceted strategy did not have a statistically significant effect on reducing the fall and injurious fall rates. In other words, education on nurses may not impact fall occurrences. Therefore, it is proposed that nurses' attitudes may be considered as potential for intervention.

The nursing society often assumes that setting an educational goal for nurses will both increase their knowledge and change their practice. If nursing is a profession, nurses working in different regions, cultures, and countries and using different levels of medical technology and equipment for patient care should practice standardized procedures competently (e.g., preventing hospital-acquired injurious falls). Professional care quality standards (e.g., skills and clinical judgment) should be universal (Tzeng \& Yin, 2008b).

In addition, it seems that the society in general, including the nursing society, commonly assumes that nurses treat patients as well as they would their own families. It is possible that cultivating and sustaining a caring attitude among clinicians is often overlooked as an intervention strategy. Therefore, it is proposed that noncaring nurses can be one of the causes of patient falls. A caring attitude refers to clinicians' approaches to administering care. Attitude refers to a mental state of readiness to respond in a characteristic way to a stimulus (e.g., an object, concept, or situation) (Merriam-Webster OnLine, 2010).

\section{Purpose and Significance of This Paper}

The purpose of this article is to first discuss the barriers to implementing fall prevention programs in acute care hospitals. The attributional theory of success and failure is used to analyze these barriers, and the analysis is followed by a discussion of whether there is a formula to ensure successful implementation. Additionally, the author considers whether a lack of knowledge and/or a lack of caring attitude play a role as the underlying barriers to implementing a successful fall prevention program. Possible educational strategies as interventions in fall prevention programs in acute hospital settings are also discussed. Finally, a patient's story illustrates patients' expectations for a care environment centered on their needs. This article is significant because, since October 2008, Medicare 
has no longer reimbursed acute care hospitals for the costs of additional care required due to hospitalacquired injuries (e.g., injurious falls) (Centers for Medicare \& Medicaid Services [CMS], 2008).

\section{Barriers to Implementing Fall Prevention Programs in Acute Care Hospitals}

Results of translating research evidence into practice to change clinicians' behaviors have been inconclusive. Interventions may be effective in one study, but not be in others. Multifaceted interventions (e.g., reminders, education sessions, and barrier-oriented interventions) did not appear to be more effective than a single intervention, particularly reminders (Dijkstra et al., 2006; Grimshaw et al., 2001, 2004; Titler, 2007).

In an Australian study, Fonda, Cook, Sandler, and Bailey (2006) claimed that successful implementation of a fall prevention program with sustained reduction in serious fall-related injuries in older people in a hospital was mediated by strong leadership and environmental support that ensured a practice environment that was conducive to change. However, a review study conducted by Dijkstra et al. (2006) led to a different conclusion. They found that organizational factors (management support, functional differentiation and specialties, and guideline implementation interventions developed within a hospital versus derived from sources outside a hospital) were not significantly associated with the effectiveness of implementation interventions and had no effects on improving the quality of care at hospitals. The effects of structure measures are inconclusive.

A study conducted by Koh et al. (2009) in two acute care hospitals in Singapore showed that nurses perceived the barriers to fall prevention program implementation as being related to a lack of knowledge. However, increased knowledge on fall prevention via educational interventions did not guarantee effective program implementation. Another study conducted by Koh, Manias, Hutchinson, Donath, and Johnston (2008) in five acute care hospitals in Singapore also concluded that knowledge was the barrier most frequently mentioned by nurses to implementation of a fall prevention clinical practice guideline. The four other greatest barriers were availability of support staff, access to equipment (e.g., bed alarm devices and night lights), health status of patients, and education of staff. It was noted that nurses' attitude and their perceived role were only ranked as the llth frequently mentioned barrier.

The locus-stability classification scheme in the attributional theory of success and failure (Weiner, 1986; Weiner et al., 1971) was used to analyze the five barriers most frequently identified as being external versus internal and being stable or unstable from the point of view of nurses (Koh et al., 2008, 2009). Weiner and associates (Weiner, 1986; Weiner et al., 1971) used a locus-stability classification scheme (a $2 \times 2$ categorization scheme; internal and external control orientations and whether or not they were stable) to characterize the perceived causes of achievement-related context (success or failure) (Table 1). This locus-stability classification scheme is represented as a $2 \times 2$ window with four cells. The four contexts identified in this scheme were (1) aptitude (internal, stable), (2) temporary exertion (internal, unstable), (3) objective task characteristics (external, stable), and (4) chance (external, unstable). The study conducted by Tzeng and Yin (2008a) is an example of using Weiner and associates' (Weiner, 1986; Weiner et al., 1971) locus-stability classification scheme paired with incident reports and the perspectives of nurses and nursing attendants to understand the risk factors that contribute to inpatient falls.

The top five barriers to fall prevention program implementation identified in the study conducted by Koh et al. (2008) were categorized as being external and being unstable (two barriers: difficulties with availability of support staff and health status of patients), being external and being stable (one barrier: access to facilities), and being internal and being stable (two barriers: a lack of knowledge and education of staff) from nurses' perspectives.

However, in order to address the barriers associated with a lack of knowledge and the education of staff,

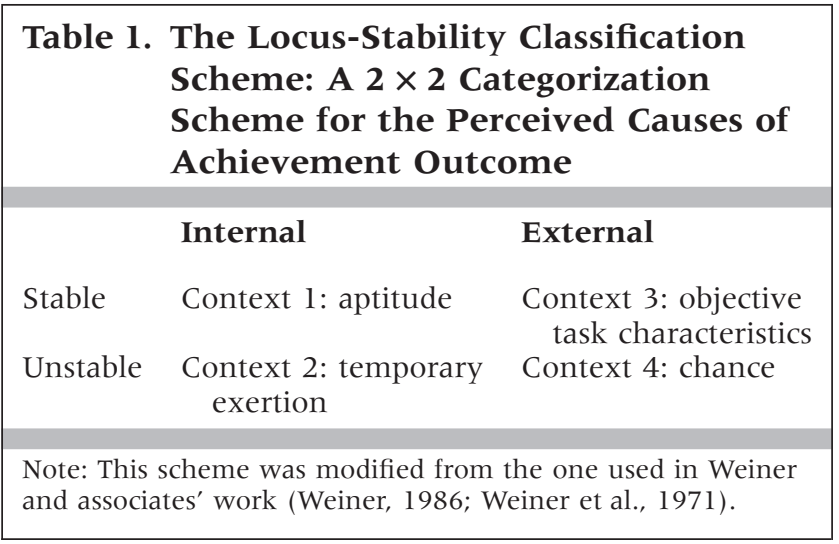


healthcare organizations need to provide educational interventions. Therefore, a lack of knowledge and the education of staff may also be categorized as being external and unstable, because the frequency and depth of fall prevention-related in-service training may vary depending on the resources available for training and the leadership commitment to training. If so, all barriers may be attributed to external factors (e.g., structure measures). The outcomes of the educational strategies may also vary due to factors of temporary exertion (e.g., nurses are too tired to comprehend the materials).

It is unclear why increased knowledge about fall prevention via educational interventions does not guarantee effective implementation of a fall prevention program (Koh et al., 2009). Is nurses' adherence to guidelines due to a lack of facilities and equipment or to a lack of patient-centered care (e.g., developing an individualized care plan on fall prevention based on the risk factors of each individual patient)? Based on Weiner and associates' (Weiner, 1986; Weiner et al., 1971) locus-stability classification scheme, nurses' attitudes would be categorized as being internal and stable. In order to change nurses' caring attitude for providing patient-centered fall prevention care, in-service training and school nursing education may need to focus on cultivating and strengthening an attitude that centers on patients. However, no studies have investigated the link between nurses' caring attitude and patient outcomes.

\section{Is There a Formula to Ensure Successful Implementation?}

It is arguable whether a simple formula exists to ensure successful implementation of research-based clinical improvements. Regardless, when designing an effective implementation strategy, it is necessary to target individual healthcare institutions' structural and attitudinal needs and the barriers to and facilitators of behavior change. Interventions may be targeted at either the structure or the process of care or at both (Grol, 1997; Grol \& Grimshaw, 1999). However, the existence of research evidence alone may not be enough to influence nurses' shared values, beliefs, and behavioral norms toward patient safety (e.g., preventing hospital-acquired injuries), and to change practices and the work environment (e.g., behaviors of both clinical professionals and managers) (Aberg et al., 2009; Feng, Bobay, \& Weiss, 2008).

\section{Is There a Lack of Knowledge or a Lack of Caring Attitude?}

Reflecting on the study conducted by Koh et al. (2009), in which increased knowledge on fall prevention via educational interventions did not guarantee effective fall prevention program implementation, brought up several principal questions. Why doesn't nursing education on fall prevention result in better outcomes? Is it possible that a lack of nurses' caring attitudes is the underlying cause (e.g., providing patient-centered care), instead of a lack of professional skills and knowledge (e.g., how to implement a fall prevention protocol)?

As a matter of fact, fall prevention principles (e.g., eliminating extrinsic risk factors and intrinsic risk factors) often apply to hospitalized patients and community residents (Currie, 2008). Are most of the well-known interventions and risk assessment guides in the fall prevention protocol common sense? If so, a lack of knowledge may not be an underlying barrier to effective implementation of a fall prevention program.

\section{Discussion and Practical Implications: Rethink Approaches for Fall Prevention Interventions}

A successful strategy may need to equally address two education goals: (a) promotion of nurses' professional knowledge and skills in implementing a fall prevention program, and (b) cultivation of nurses' attitudes in treating patients as their own families. Setting educational goals with a focus on nurses' attitudes may result in a change in nursing practice, and the sustained change may support a patient-centered and relationship-based practice environment (Koloroutis, 2004; McCormack \& McCance, 2006). The following story of a dissatisfied patient illustrates the desperate need to support a patient-centered and relationship-based practice environment.

Within the past 6 years, a 57-year-old White man, employed as a licensed lawyer and administrator in an academic setting with a medical center, had prostate surgery with two post-surgery hospital readmissions through the emergency room as well as an outpatient out-of-pocket eye surgery to correct myopia at the same medical center he was employed. The eye surgery had a disappointing result and was repeated, but the result remained unsatisfactory. 
Nevertheless, he did not file any lawsuits against his employer. He stated that he stays with his doctors and the medical center affiliated with his employer because he thinks that his hospital provides stateof-the-art medical care, or at least it is supposed to. He did have one outpatient surgery in the same hospital for treatment of blood veins in his leg with a very satisfactory result.

He said: "The inpatient and outpatient care I experienced was not personal. My hospital only considered me as a case, not a person. The doctors and nurses were not concerned enough about my needs. ... When I was hospitalized, I was able to understand and remember my doctors' orders, but nurses were not following the orders. I used call lights to remind my nurses, but they ignored me. They just did not try hard enough. ... I remembered that when my four children were born in the other hospital, the atmosphere was so warm, nice, and pleasant. Maybe such a pleasant atmosphere only exists in the childbirth center."

\section{Reflection on the Story: Patients Expect a Patient-Centered Care Environment}

If nurses and doctors treated their patients as well as they would their own families, patient-centered care would be well perceived. Caring is indeed the essence of nursing practice. In acute care settings, patients are often treated as diagnoses instead of patients, especially in large institutions. Hospital rules and policies are there to serve the needs of the organization itself more than the needs of the patients and their families. In the healing process, effective nursing requires elements of technical expertise and compassion as well as a care environment that enhances the use of patientcentered process (McCormack \& McCance, 2006; Moriconi \& Stabler-Haas, 2010; Weydt, 2010).

In Watson's (1999) theory of human caring, four imperatives for caring were identified: (a) honoring the dignity and worth of each person, (b) recognizing the individual's unique response to illness, (c) protecting the individual's autonomy, and (d) helping individuals reach their maximum capacity. Swanson (1993) depicted five essential caring processes: maintaining belief, knowing, being with, doing for, and enabling and informing. Koloroutis's (2004) relationship-based care model described a caring and healing environment in which each individual is seen as a whole person (body, mind, and spirit). Continuity of nurse-patient and nurse-family relationships is the foundation of quality care delivery (Koloroutis, 2004).

Patient-centered and relationship-based care envisions education as a process that incorporates caring and mind-body connections, as well as respect for patients' differences, preferences, and expressed needs (Moriconi \& Stabler-Haas, 2010). This process is meant to help nurses comprehend patient care, which includes the relationships of nurse to self, nurse to patient, and nurse to colleagues, and to revolutionize patient-centered care in a cost-effective manner. In other words, clinical competence, critical thinking, caring, compassion, respect, and incorporation of the mind-body connection should be the components of every nursing curriculum. However, incorporating the body-mind-spirit connection into the curriculum remains a challenge to nurse educators (Moriconi $\delta$ Stabler-Haas, 2010).

As an extension to McCormack and McCance's (2006) framework for person-centered nursing, it is arguable whether treating patients as well as one's own families should be one of the attributes of a nurse. To cultivate and sustain a caring attitude among clinicians, it is essential to have a caring culture in the workplace, including the quality and commitment of leadership and clinicians' and administrators' attitude of treating each other as well as their own families. It could be argued that a practice environment with a caring attitude to colleagues should come first.

\section{Nurses' and Patients' Understanding About Patient-Centered Care}

In the study by McCance, Slater, and McCormack (2009), nurses had a clear idea of what caring and nursing consisted of and that was reflected in patientcentered care. However, introducing patient-centered nursing as a practice development intervention into the inpatient setting of a tertiary hospital had no effect on nurses' perceptions about patient-centered care as measured by their agreement levels to the question "Do you consider the following aspects of your nursing practice to be caring?" In contrast, patients' perceptions of caring varied before and after the patient-centered nursing practice intervention was introduced. However, no patient-centered or clinical outcome measures were investigated in this study.

McCance et al.'s (2009) study suggested that nurses have a solid understanding about caring, but they may not demonstrate the appropriate caring attitudes when they encounter their patients. Maybe some nurses 
simply do not walk the talk-that is, they understand the meaning of caring but do not perform patientcentered care with a caring attitude. Caring attitude should be among professional nursing competencies and indeed has been assumed to be a given attitude upon graduation or licensure as a nurse. If so, measuring patients' perceptions about the care they receive during hospital stays may be a way to assess nurses' caring attitudes as expected and perceived by patients.

In a recently developed Person-Centered Climate Questionnaire-patient version (Edvardsson, Koch, \& Nay, 2009; Edvardsson, Sandman, \& Rasmussen, 2008), the person-centered climates comprise safety, everydayness, and hospitality in the Swedish version, and safety and hospitality in the English version. As a matter of fact, the CMS in the United States has mandated that hospitals measure nurses' and doctors' attitudes. On the Hospital Compare Web site supported by CMS, Hospital Consumer Assessment of Healthcare Providers and Systems (http://www.hospitalcompare. hhs.gov/hospital-search.aspx?AspxAutoDetectCookie Support=l and http://www.cms.gov/HospitalQuality Inits/16_InpatientMeasures.asp\#TopOfPage) results are reported for six summary measures, two individual items, and two global ratings, a total of 10 measures (CMS, 2009).

The six summary measures encompass (a) how well nurses communicate with patients, (b) how well doctors communicate with patients, (c) how responsive hospital staff are to patients' needs, (d) how well hospital staff help patients manage pain, (e) how well the staff communicates with patients about medicines, and (f) whether key information was provided at discharge. The two individual items address the cleanliness and quietness of patients' rooms, and the two global ratings report patients' overall rating of the hospital and whether they would recommend the hospital to family and friends (CMS, 2009).

\section{Reflection on the Story: Are Nurses and Doctors Working to Avoid Lawsuits?}

The patient who shared with his story with me also claimed that his nurses and doctors seemed to have been working simply to avoid lawsuits. One question relating to the attitude of nurses and doctors was brought up by the patient himself: Are nurses and doctors interested in their patients? He responded "no" without any hesitation. In a very firm tone, he expressed that nurses and doctors are working only to avoid lawsuits.
Two questions rose while reflecting on my conversation with the patient. Are we assuming that nurses are providing patient-centered care within a relationship-based practice environment? Are we assuming that nurses are delivering holistic care with emphases on their patients' body (by initiating meaningful and concrete conversations for understanding and addressing physical needs), mind (by initiating effective and open conversations for understanding and addressing their emotional needs), and spirit (by initiating mutual healing conversations for understanding and addressing their spiritual needs) (Koloroutis, 2004)? From the standpoint of nursing society as a whole, the answers to these questions should be yes.

Based on the information presented, the author developed a theoretical patient-centered nursing model (Figure 1). This model is based on the personcentered nursing framework developed by McCormack and McCance (2006). Figure 1 has not been tested, and more research is needed to validate the interwoven relationships between the care environment (structure measures), nurses' professional proficiencies (structure measures), caring process (process measures), and patient outcomes (outcome measures), especially for the use of evaluating implementation of fall prevention programs on reducing fall rates.

\section{Conclusion}

Patient centeredness is a common phrase in the literature related to nursing and health care. Increasingly, there is an expectation that clinicians use the principles of patient-centered and relationship-based care in their practice and that healthcare organizations adopt the same principles by respecting the values of the service user (McCormack, 2003). Using fall prevention efforts in acute care settings as an example, good practice refers to clinicians using practice knowledge and determining the right thing to do at the right time and in the right way (e.g., applying the appropriate fall prevention interventions to each individual patient based on his or her unique needs and risk factors for falls). Good practice is seen as being clinically effective (e.g., a reduction in the injurious fall rates), which involves more than the application of a theory or the exercise of a skill (McCormack, 2003).

In the process of implementing a fall prevention program in acute care settings (as well as in other 
Figure 1. The Theoretical Patient-Centered Nursing Model. This model is based on the person-centered nursing framework developed by McCormack and McCance (2006) and is a modified one. The hierarchical order of the three ovals (patient outcomes, professional proficiencies for providing patient-centered care, and the care environment) is different from the one developed by McCormack and McCance (2006). The caring process includes five approaches (in five callouts): working with the patient's beliefs and values, providing for physical needs, having a sympathetic presence, sharing decision making with the patient, and engaging with the patient.

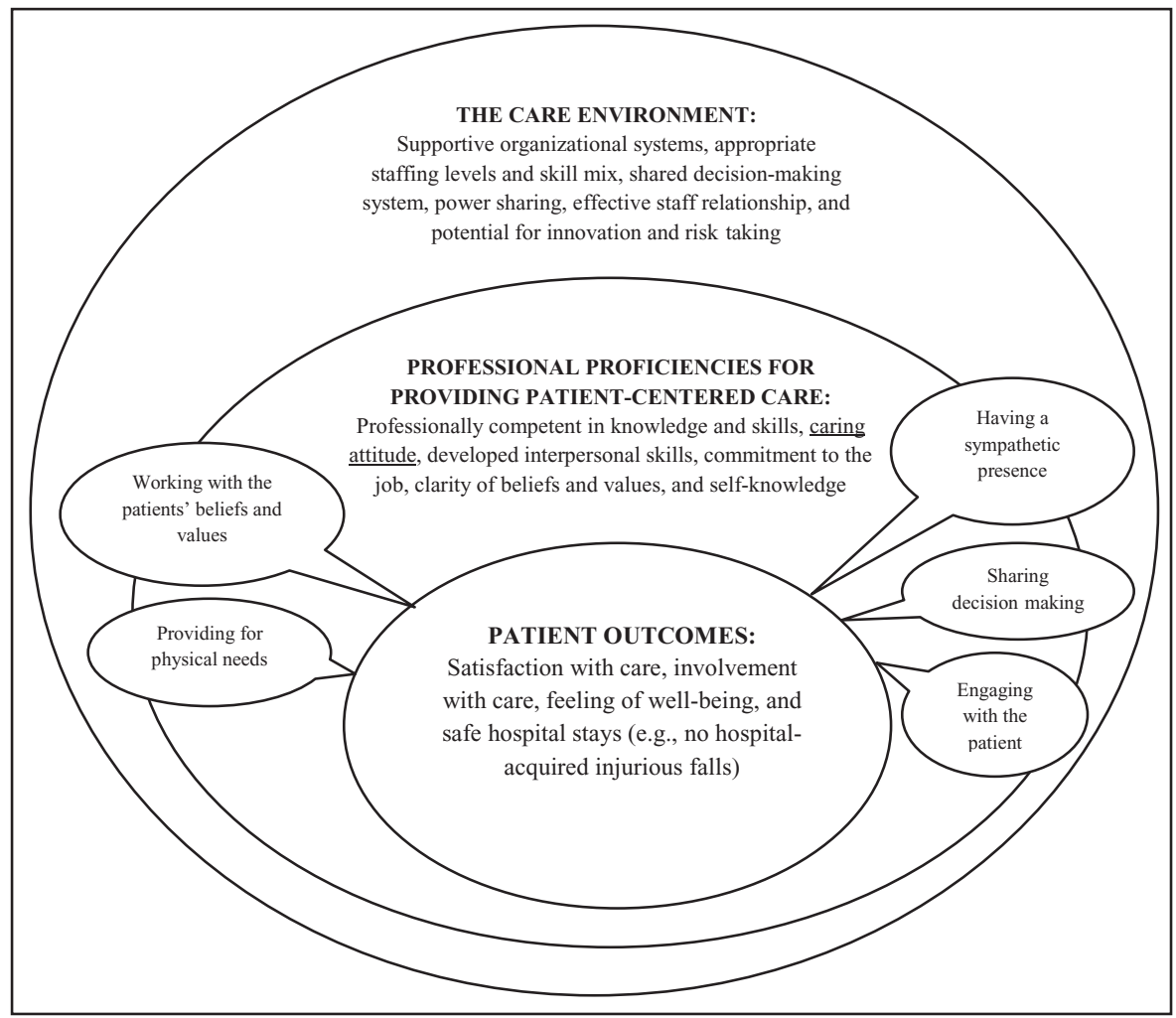

types of healthcare settings), the education goals for nurses need to not only promote their professional knowledge and skills in implementing the program, but also cultivate their caring attitudes of treating patients like their own families-the principles of providing patient-centered and relationship-based case at bedside. In addition, more research on good practice using patient-centered and relationship-based care is needed. Such research requires researchers to understand and work with individual beliefs, values, wants, needs, and desires held by others (patients, families, and colleagues in the interdisciplinary team) in order to treat patients as individual persons. Also, such research requires researchers to adopt approaches that enable flexibility, mutuality, respect, and getting along with others (McCormack, 2001, 2003). One of the ultimate goals of this line of research is to promote safer hospital stays by preventing injurious falls in acute care settings.

Visit the Nursing Forum blog at http://www. respond2articles.com/NF/ to create, comment on, or participate in a discussion.

\section{References}

Aberg, A. C., Lundin-Olsson, L., \& Rosendahl, E. (2009). Implementation of evidence-based prevention of falls in rehabilitation units: A staff's interactive approach. Journal of Rehabilitation Medicine, 41, 1034-1040.

Aiken, L. H. (2008). Economics of nursing. Policy, Politics $\theta$ Nursing Practice, 9, 73-79. 
Centers for Medicare \& Medicaid Services. (2008). Changes to the hospital inpatient prospective payment systems and fiscal year 2009 rates. Baltimore, MD: Centers for Medicare $\&$ Medicaid Services. Retrieved May 14, 201 1, from http:// www.cms.hhs.gov/AcuteInpatientPPS/downloads/CMS1390-F.pdf

Centers for Medicare \& Medicaid Services. (2009). HCAHPS fact sheet. Baltimore, MD: Centers for Medicare $\delta$ Medicaid Services. Retrieved May 14, 2011, from http://hcahps.org/files/HCAHPS \% 20Fact\%20Sheet, \%20revised 1,\%203-31-09.pdf

Currie, L. (2008). Fall and injury prevention. In R. G. Hughes (Ed.), Patient safety and quality: An evidence-based handbook for nurses (pp. 195-250). AHRQ Publication No. 08-0043. Rockville, MD: Agency for Healthcare Research and Quality, US Department of Health and Human Services.

Dijkstra, R., Wensing, M., Thomas, R., Akkermans, R., Braspenning, J., Grimshaw, J., \& Grol, R. (2006). The relationship between organisational characteristics and the effects of clinical guidelines on medical performance in hospitals, a meta-analysis. BMC Health Services Research, 6, 53. Retrieved May 14, 2011, from http://www.ncbi. nlm.nih.gov/pmc/articles/PMC 1479332/pdf/1472-69636-53.pdf

Edvardsson, D., Koch, S., \& Nay, R. (2009). Psychometric evaluation of the English language Person-Centered Climate Questionnaire-Patient version. Western Journal of Nursing Research, 31, 235-244.

Edvardsson, D., Sandman, P. O., \& Rasmussen, B. (2008). Swedish language Person-Centred Climate Questionnaire-Patient version: Construction and psychometric evaluation. Journal of Advanced Nursing, 63, 302-309.

Feng, X., Bobay, K., \& Weiss, M. (2008). Patient safety culture in nursing: A dimensional concept analysis. Journal of Advanced Nursing, 63, 310-319.

Fonda, D., Cook, J., Sandler, V., \& Bailey, M. (2006). Sustained reduction in serious fall-related injuries in older people in hospital. Medical Journal of Australia, 184, 379382.

Gilewski, M. J., Roberts, P., Hirata, J., \& Riggs, R. (2007). Discriminating high fall risk on an inpatient rehabilitation unit. Rehabilitation Nursing, 32, 234-240.

Grimshaw, J. M., Shirran, L., Thomas, R., Mowatt, G., Fraser, C., Bero, L., . . . O'Brien, M. A. (2001). Changing provider behavior: An overview of systematic reviews of interventions. Medical Care, 39, 2-45.

Grimshaw, J. M., Thomas, R. E., MacLennan, G., Fraser, C., Ramsay, C. R., Vale, L., .. . Donaldson, C. (2004). Effectiveness and efficiency of guideline dissemination and implementation strategies. Health Technology Assessment (Winchester, England), 8, iii-iiv, 1-72.

Grol, R. (1997). Personal paper. Beliefs and evidence in changing clinical practice. British Medical Journal, 315, 418-421.

Grol, R., \& Grimshaw, J. (1999). Evidence-based implementation of evidence-based medicine. The Joint Commission Journal on Quality Improvement, 25, 503-513.

Gutierrez, F., \& Smith, K. (2008). Reducing falls in a definitive observation unit: An evidence-based practice institute consortium project. Critical Care Nursing Quarterly, 31, 127-139.

Hitcho, E. B., Krauss, M. J., Birge, S., Claiborne Dunagan, W., Fischer, I., Johnson, S., . . . Fraser, V. J. (2004). Characteristics and circumstances of falls in a hospital setting: A prospective analysis. Journal of General Internal Medicine, 19, 732-739.

Joint Commission. (2005). Defining the problem of falls. In I. J. Smith (Ed.), Reducing the risk of falls in your health care organization (pp. 13-27). Oakbrook Terrace, IL: Author.

Koh, S. L., Hafizah, N., Lee, J. Y., Loo, Y. L., \& Muthu, R. (2009). Impact of a fall prevention programme in acute hospital settings in Singapore. Singapore Medical Journal, $50,425-432$.

Koh, S. S., Manias, E., Hutchinson, A. M., Donath, S., $\delta$ Johnston, L. (2008). Nurses' perceived barriers to the implementation of a fall prevention clinical practice guideline in Singapore hospitals. BMC Health Services Research, 8, 105. Retrieved May 14, 2011, from http:// www.ncbi.nlm.nih.gov/pmc/articles/PMC2422837/pdf/ 1472-6963-8-105.pdf

Koloroutis, M. (2004). Relationship-based care: A model for transforming practice. Minneapolis, MN: Creative Health Care Management.

McCance, T., Slater, P., \& McCormack, B. (2009). Using the caring dimensions inventory as an indicator of personcentred nursing. Journal of Clinical Nursing, 18, 409417.

McCormack, B. (2001). Negotiating partnerships with older people: A person-centred approach. Aldershot, UK: Ashgate Press.

McCormack, B. (2003). Researching nursing practice: Does person-centredness matter? Nursing Philosophy, 4, 179188.

McCormack, B., \& McCance, T. V. (2006). Development of a framework for person-centred nursing. Journal of Advanced Nursing, 56, 472-479.

Merriam-Webster OnLine. (2010). Attitude. Springfield, MA: Merriam-Webster. Retrieved May 22, 2010, from http:// www.merriam-webster.com/dictionary/attitude

Moriconi, C., \& Stabler-Haas, S. (2010). Making connections: Integration of psychiatric and medical-surgical nursing and relationship-based care. Creative Nursing, 16, 13-17.

Nadkarni, J. B., Iyengar, K. P., Dussa, C., Watwe, S., \& Vishwanath, K. (2005). Orthopaedic injuries following falls by hospital in-patients. Gerontology, 51, 329-333.

Quigley, P. A., Hahm, B., Collazo, S., Gibson, W., Janzen, S., Powell-Cope, G., ... White, S. V. (2009). Reducing serious injury from falls in two veterans' hospital medical-surgical units. Journal of Nursing Care Quality, 24, 33-41.

Raeder, K., Siegmund, U., Grittner, U., Dassen, T., \& Heinze, C. (2010). The use of fall prevention guidelines in German hospitals-A multilevel analysis. Journal of Evaluation in Clinical Practice, 16, 464-469.

Rizzo, J. A., Friedkin, R., Williams, C. S., Nabors, J., Acampora, D., \& Tinetti, M. E. (1998). Health care utilization and costs in a Medicare population by fall status. Medical Care, 36, 1174-1188. 
Schwendimann, R., Bühler, H., De Geest, S., \& Milisen, K. (2006). Falls and consequent injuries in hospitalized patients: Effects of an interdisciplinary falls prevention program. BMC Health Services Research, 6, 69. Retrieved May 14, 2011, from http://www.ncbi.nlm.nih.gov/pmc/ articles/PMC 1534028/pdf/1472-6963-6-69.pdf

Swanson, K. (1993). Nursing as informed caring for the well-being of others. Journal of Nursing Scholarship, 25, 352-357.

Tinetti, M. E., Inouye, S. K., Gill, T. M., \& Doucette, J. T. (1995). Shared risk factors for falls, incontinence, and functional dependence. Unifying the approach to geriatric syndromes. Journal of the American Medical Association, 273, 1348-1353.

Tinetti, M. E., \& Kumar, C. (2010). The patient who falls: "It's always a trade-off." Journal of the American Medical Association, 303, 258-266.

Tinetti, M. E., \& Williams, C. S. (1997). Falls, injuries due to falls, and the risk of admission to a nursing home. New England Journal of Medicine, 337, 1279-1284.

Titler, M. (2007). Translating research into practice. American Journal of Nursing, 107(6 Suppl.), 26-33.
Tzeng, H. M., \& Yin, C. Y. (2008a). The extrinsic risk factors for inpatient falls in hospital patient rooms. Journal of Nursing Care Quality, 23, 233-241.

Tzeng, H. M., \& Yin, C. Y. (2008b). What's our philosophy? Journal of Professional Nursing, 24, 5-6.

Tzeng, H. M., \& Yin, C. Y. (2009). Inpatient falls: The impact of family and personal caregivers. Applied Nursing Research, 22, 159-165.

Tzeng, H. M., Yin, C. Y., \& Grunawalt, J. (2008). Effective assessment of use of sitters by nurses in inpatient care settings. Journal of Advanced Nursing, 64, 176-183.

Watson, J. (1999). Postmodern nursing and beyond. St. Louis, MO: Elsevier.

Weiner, B. (1986). An attributional theory of motivation and emotion. New York: Springer-Verlag.

Weiner, B., Frieze, I. H., Kukla, A., Reed, L., Rest, S., \& Rosenbaun, R. M. (1971). Perceiving the causes of success and failure. Morristown, NJ: General Learning Press.

Weydt, A. (2010). Mary's story, relationship-based care delivery. Nursing Administration Quarterly, 34, 141-146. 